\title{
Study on Damage Constitutional Relation and Attenuation Law of Elastic Wave in Fractured Rock Mass
}

\author{
Hang Zhao ${ }^{1,2, a}$, Xinping $\mathrm{Li}^{1, \mathrm{~b}}$ and Qian Dong ${ }^{1,2}$ \\ ${ }^{1}$ Hubei Key Laboratory of Roadway Bridge \& Structure Engineering, Wuhan University of \\ Technology, Wuhan, China \\ ${ }^{2}$ School of Civil Engineering \& Architecture, Wuhan University of Technology, Wuhan, China \\ a525818527@qq.com
}

Keywords: High stress, Similarity theory, Triaxial compression, Wave velocity, Confining pressure

\begin{abstract}
Fissures in the high stress mass of deep buried had a great influence on mechanical properties and wave velocity of rock mass. Based on similarity theory, Rock like materials simulated deep buried marble of Jinping-II Hydropower Station. Under different fracture and different confining pressure, the triaxial compression test could be used in the model of the specimen. Collected the data timely and plotted the stress-strain curve and the stress-acoustic curve in the process of tests, then the model test-piece was equivalent to transversely isotropic material with multi group uniform distribution crack, established the damage constitutive relation which contains the acoustic wave velocity variable. The research achievement shows: In the triaxial compression test, the wave velocity increases with the axial compression when confining pressure is constant, the text advanced the speed by the first fast then slow way, the wave velocity increases with the axial compression is constant; the angle of fracture has a significant influence on acoustic wave velocity and attenuation of the energy of acoustic wave, under the same stress state, the larger the angle between the propagate direction of velocity and the fracture, the attenuation of sonic energy is more obvious, and the smaller the acoustic velocity.
\end{abstract}

\section{Introduction}

The existence of joints has great influence on the physical and mechanical properties of rock mass, the energy attenuation characteristics and wave velocity of elastic wave are changed. So the study of propagation and attenuation law of elastic wave in deep rock mass with joints is full of meaning.

Many scholars and experts have studied the propagation and attenuation of elastic waves in fractured rock mass. In 1973,R.E.Thill[1] studied the relationship between the change of elastic wave velocity and the joints in rock. In 1997,R. Holt [2] got the wave velocity of elastic wave in the rock mass with different crack distribution through laboratory tests. In china, the research on the laws of the elastic wave propagation in rock date back from 1970.Gao longsheng and Ge huancheng[3] test the elastic wave velocity at different confining pressure which include ten rock samples. Huang chengxian and Song dawei[4] test the changing rule of the elastic wave velocity of rock in different confining pressure; Wang hongtu [5] etc. studied the dynamic elastic parameters of the fractured rock mass, the relationship between the elastic wave velocity and fracture. Zhao mingjie[6]studied the relationship among the elastic wave velocity, attenuation and stress based on the bearing and deforming of joint.Liu yonggui[7]etc. studied the reflective relationship when elastic wave go through the joint surface of basalt.

In conclusion, scholars at home or abroad put lots of effort into the correlation between stress effect, crack distribution and wave velocity and the propagation attenuation of sonic waves in fractured rock mass, and lots of achievements have been obtained as well. However, there are less reports on the study of the elastic wave propagation of deep fractured rock mass under different stress paths at present. Because of the complicated existing environment of deep fractured rock mass, the field sonic wave test and sampling of deep fractured rock mass are extremely hard. Therefore, through laboratory model experiments, triaxial compressive tests were conducted on plaster models corresponding to deep fractured rock mass in this paper. This paper has also made real-time 
monitoring and system analysis on stress, strain and sonic wave velocity, and the constitutive relation of damaged fractured rock including sonic wave velocity variables was derived as well.

\section{Experiment scheme}

The model test is carried out on the three axis test machine, as is shown in Fig.1, at the same time, use the ultrasonic testing system to conduct data acquisition and transmission parameters. Before the test, in the key part of the specimen, the vertical and horizontal strain of the paste is pasted symmetrical, and fixed the elastic wave test probe, the experiments were conducted in a quiet environment.

According to the excavation of deep buried tunnel in Hydropower Station, the stress path of the three direction loading is determined, in triaxial compression test:(1).At the same time, loading the $\sigma_{2}, \sigma_{3}$ to set value $(0.6 \mathrm{MPa}, 1.2 \mathrm{MPa}, 1.8 \mathrm{MPa})(2)$. Keep $\sigma_{2}, \sigma_{3}$ unchanged, loading $\sigma 1$ until sample damage. In the test, the stress loading rate is kept constant. the dimensions and loading modes of the model are shown in the following diagram, as is shown in Fig.2.

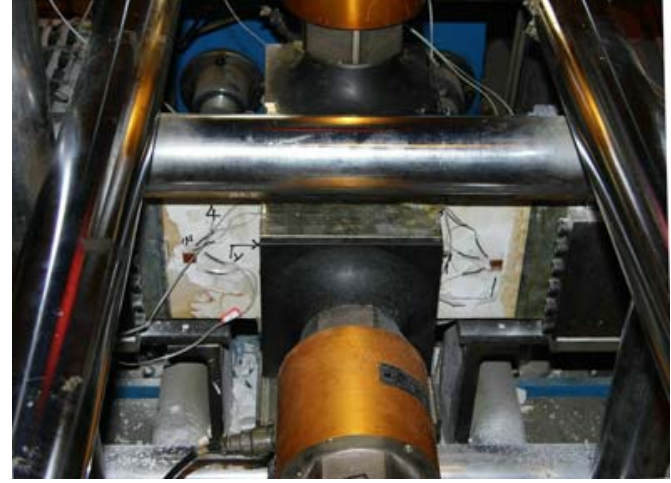

Fig.1 the true triaxial test system

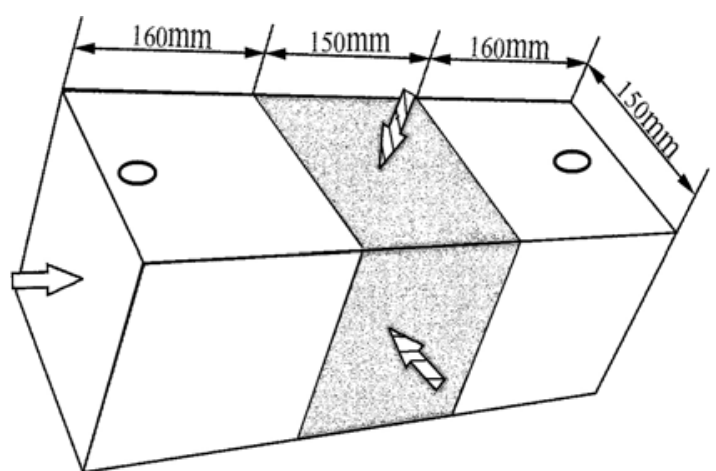

Fig.2 model of the specimen

\section{Experimental results and analysis}

Based on the data collected from the triaxial compression test, the stress-wave velocity curve of the elastic wave in the fractured rock mass is got. Take the elastic wave testing of double fractured rock mass as an example,Figure. 3 is the axial compression-wave velocity curve under under different confining pressures,Figure. 4 is the axial compression-wave velocity curve under under different crack angles,Figure. 5 is the axial compression-wave velocity curve under under different fracture number.

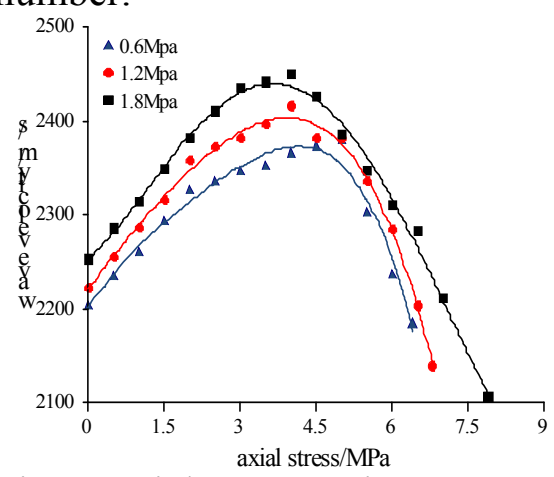

Fig.3 Axial compression-wave velocity curve

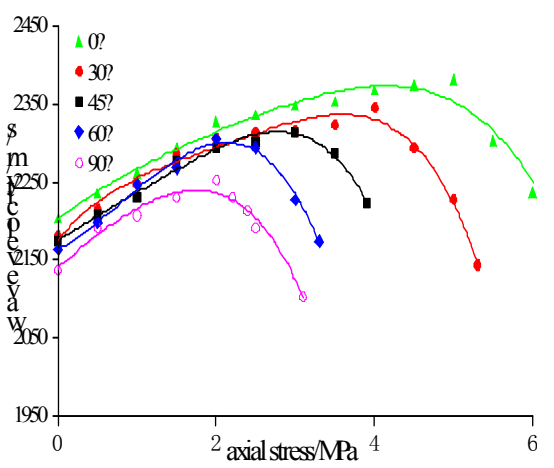

Fig.4 Axial compression-wave velocity curve

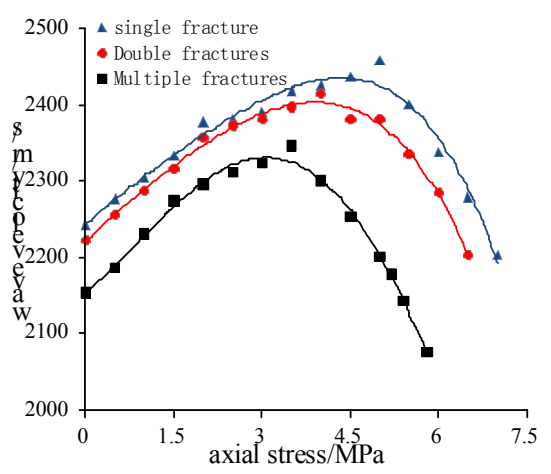

Fig.5 Axial compression-wave velocity curve

As shown in Figure 3.With the constant confining pressure and increasing axial compression pressure, there are six phases for the change of elastic wave velocity: initial wave velocity $\rightarrow$ rapid increase $\rightarrow$ slow increase $\rightarrow$ basic stability $\rightarrow$ peak wave velocity $\rightarrow$ peak wave velocity $\rightarrow$ rapid decline. This is due to the axial stress causes the opening and closing of cracks in rock mass, and resulting in the change of the elastic wave velocity value: at the end stage of loading, when the axial stress reaches 
$60 \%$ of its maximum intensity, the velocity rapidly reduces, showing that this is the starting point for the rock to expand.

As the constant compression pressure and increasing axial confining pressure:(1)At the initial stage, fractured rock mass is only by confining pressure, the greater the confining pressure, the greater the volume of crack closure in rock mass.(2)At the intermediate stage, the effect of confining pressure limits the volume change of crack in rock mass, the more the confining pressure, the greater the restriction, before the peaks of the wave velocity value, the greater the confining pressure, the slower the growth rate of the elastic wave velocity.(3)At the final stage, comparing to the axial pressure, the confining pressure weakening, micro cracks begin to run through, so after the peak of the wave velocity, the smaller the confining pressure, the faster the wave velocity is decreased.

As shown in Figure 4.(1)At the initial stage, fractured rock mass is only confined to confining pressure, with the increase of crack angle, the elastic wave velocity decreases.(2)In the whole process of testing, in the same stress along with the increase of the fracture angle, the elastic wave velocity values show a downward trend, the influence of crack angle on the elastic wave velocity is mainly by the projection of the fracture area, the larger the projection area, the faster wave velocity value decline.

As shown in Figure 5.With the increase of the number of cracks, the wave velocity decreases, when the fractured rock mass under the same stress level, the gap between wave velocity in rock mass with two cracks and wave velocity in rock mass with single crack is small, the average decline in the value of wave velocity is about $30 \mathrm{~m} / \mathrm{s}$, but wave velocity in rock mass with multiple cracks decreased significantly, the average decline in the value of wave velocity is about $120 \mathrm{~m} / \mathrm{s}$, This is due to the increase of the number of cracks in the unit volume greatly reduce the integrity of the specimen, so as to the decrease of elastic wave velocity value in the specimens.

Combined the curve of compression-wave velocity, the model material can be considered as a homogeneous material in a single direction, considering the damage of the material in a single direction, so introducing damage variable $\mathrm{Di}$,In order to describe the damage degree of unidirectional material under any condition of the loading process, as shown in Table 1:

$$
\left\{\begin{array}{l}
\sigma_{x}=\left(1-D_{x}\right)\left(c_{11} \varepsilon_{x}+c_{12} \varepsilon_{y}+c_{13} \varepsilon_{z}\right) \\
\sigma_{y}=\left(1-D_{y}\right)\left(c_{12} \varepsilon_{x}+c_{11} \varepsilon_{y}+c_{13} \varepsilon_{z}\right) \\
\sigma_{z}=\left(1-D_{z}\right)\left(c_{13} \varepsilon_{x}+c_{13} \varepsilon_{y}+c_{33} \varepsilon_{z}\right) \\
\tau_{x y}=\left(1-D_{x y}\right) c_{44} \gamma_{x y} \\
\tau_{y z}=\left(1-D_{y z}\right) c_{55} \gamma_{y z} \\
\tau_{z x}=\left(1-D_{z x}\right) c_{55} \gamma_{z x}
\end{array}\right.
$$

In view of the wave velocity in the single direction of the model material, as shown in Table 2:

$$
E_{i}=\rho V_{p i}^{2}
$$

Damage variable of fractured rock mass in a single direction Can be defined by wave velocity, as shown in Table 3:

$$
D_{i}=1-\frac{V_{d i}^{2}}{V_{p f}^{2}}
$$

$v_{d i}$ is the acoustic wave velocity of I direction in the process of model test, $v_{p f}$ is the acoustic wave velocity for the complete model, $\mathrm{D}_{\mathrm{i}}$ is the damage variable in I direction.

Take table (3) into table (2), the constitutive relation can be built up with damage coupling effect: 


$$
\left\{\begin{array}{l}
\sigma_{x}=\frac{V_{d x}^{2}}{V_{p x}^{2}}\left(c_{11} \varepsilon_{x}+c_{12^{2}} \varepsilon_{y}+c_{13_{z}} \varepsilon_{z}\right) \tau_{x y}=\frac{\sqrt{V_{d x}^{2}+V_{d y}^{2}}}{\sqrt{V_{p x}^{2}+V_{p y}^{2}}} c_{44} \gamma_{x y} \\
\sigma_{y}=\frac{V_{d y}^{2}}{V_{p y}^{2}}\left(c_{12^{2}} \varepsilon_{x}+c_{11} \varepsilon_{y}+c_{13} \varepsilon_{z}\right) \tau_{y z}=\frac{\sqrt{V_{d y}^{2}+V_{d z}^{2}}}{\sqrt{V_{p y}^{2}+V_{p z}^{2}}} c_{55} \gamma_{y z} \\
\sigma_{z}=\frac{V_{d z}^{2}}{V_{p z}^{2}}\left(c_{13} \varepsilon_{x}+c_{13} \varepsilon_{y}+c_{33_{z}} \varepsilon_{z}\right) \tau_{z x}=\frac{\sqrt{V_{d z}^{2}+V_{d x}^{2}}}{\sqrt{V_{p z}^{2}+V_{p x}^{2}}} c_{55} \gamma_{z x}
\end{array}\right.
$$

\section{Conclusion}

This paper takes Jinping hydropower station as the prototype of deep buried marble, based on similarity theory, the elastic wave testing experiment of fractured rock mass under the condition of axial compression is carried out, through the contrast analysis of test results, the following main conclusions are obtained. In axial compression test, with the increase of axial stress, the value of wave velocity is increasing, as the axial compression reached $60 \%$ of the peak intensity, wave velocity value drops rapidly; the closing and opening of cracks in rock mass will cause the change of the elastic wave velocity value, the opening of the crack is more influence on the wave velocity than the closing of the crack; the confining pressure, fracture angle, the number of cracks all have an impact on the velocity of elastic wave. The degree of sensitivity of the impact in decreasing order is ranked as: the number of cracks $>$ confining pressure $>$ crack angle.

\section{Acknowledgements}

This work was financially supported by the National Natural Science Foundation of China (51274157) .

\section{References}

[1] Thill R E, Bur T R, Steckley R C.Velocity anisotropy in dry and saturated rock spheres and its relation to rock fabric[J].International Journal of Rock Mechanics and Mining Sciences \& Geomechanics Abstracts, 1973,10(6)535-557.

[2] Holt R, Furre A, Horsrud P. Stress dependent wave velocities in sedimentary rock cores: Why and why not. Int J Rock Mech Min Sci Geomech Abstr,1997, 34(3):261-276.

[3] GAO Long-shen, GE Huan-cheng. A preliminary study of p-wave and s-wave velocities under high pressure of rock samples from the mainland of China[J].Chinese Journal of Geophysics, 1975,18(1):26-38.

[4] HUANG Cheng-xian, SONG Da-wei. Study on elastic wave velocity of rock under confining pressure[J].Chinese Journal of Geotechnical Engineering, 1991,13(2): 32-41.

[5] WANG Hongtu, LI Xiaohong, YANG Chunhe, et al. The influence of cracks on the propagation properties of elastic waves in quasi-isotropic cracked rock masses[J].Rock and Soil Mechanics, 2005,26(6):873-876.

[6] ZHAO Ming-jie. Study on wave velocity and of rock mass in 2D stresses field[J]. Chinese Journal of Rock Mechanics and Engineering, 2007, 26(1):123-130.

[7] LIU Yong-gui, XU Song-lin, XI Dao-ying, et al. Dispersion effect of elastic wave in jointed basalt[J]. Chinese Journal of Rock Mechanics and Engineering, 2010, 29(S1):3314-3320. 